\title{
Relevancia del aprendizaje cooperativo sobre los diferentes perfiles de la dinámica bullying. Un análisis mediante pruebas de tamaño del efecto
}

\author{
Benito León del Barco, María-Isabel Polo del Río, Margarita Gozalo Delgado y Santiago Mendo Lázaro
}

Departamento de Psicologia y Antropología. Universidad de Extremadura. (Spain).

\begin{abstract}
Resumen: Esta investigación pretende determinar sobre qué perfil de la dinámica bullying (Agresor, Víctima, Observador) es más relevante una intervención en aprendizaje cooperativo. Mediante pruebas estadísticas de tamaño del efecto. Con el fin de evaluar las conductas de acoso se diseñó un instrumento para evaluar el acoso escolar entre Iguales desde la perspectivas del Agresor, Victima y Observador". Se trabajó con dos grupos de participantes de $3^{\text {er }}$ ciclo de primaria, un primer grupo de 311 alumnos que utilizamos para el análisis psicométrico del instrumento y un segundo grupo de 110 alumnos para la intervención. Los resultados manifiestan que el tamaño del efecto intergrupo ha sido elevado para el factor de exclusión social para los perfiles observador $(d=0.64)$ y agresor $(d=0.65)$. También, obtiene un tamaño elevado el factor agresiones verbales y físicas directas para el perfil observador $(d=0.57)$. En síntesis, tras el análisis del tamaño del efecto podemos afirmar que el aprendizaje cooperativo es eficaz sobre el perfil agresor y, especialmente, sobre el observador.
\end{abstract}

Palabras claves: Aprendizaje cooperativo, acoso entre iguales, agresor, víctima, observador, alumnos, aula.
Title: Relevance of cooperative learning about the different profiles of the bullying dynamic. An analysis by testing the effect size.

Abstract: With this research we try to determine which dynamic profile bullying (Aggressor, Victim, Observer) is more relevant in cooperative learning intervention. We will use evidence of effect size. In order to assess bullying behaviors have designed an instrument "Frequency Scale Peer Bullying. Perspective Aggressor, Victim and Observer". We have worked with two groups of participants in the 3rd cycle of primary, a first group of 311 students we used for the psychometric analysis of the instrument and a second group of 110 students for intervention. Our results show that the inter-group effect size was high for the social exclusion factor for observer profiles $(d=0.64)$ and aggressor $(d=0.65)$. Also, get a higher size factor direct verbal and physical attacks on observer profile $(d=0.57)$. In synthesis, after the analysis of the effect size we found that cooperative learning is effective on the offender profile and especially on the observe.

Key words: Cooperative learning, bullying, aggressor, victim, observer, student, classroom.

\section{Introducción}

El bullying no es un fenómeno nuevo, pero si es el más estudiado de todos cuantos pueden ocurrir en el contexto escolar, y es el que más daño moral, psicológico y educativo produce entre los escolares (Avilés, 2006). El acoso escolar recoge como protagonistas a un grupo de alumnos con papeles bien diferenciados (Avilés, 2004; Cerezo, 2006). La víctima -en la que provoca ansiedad, alteraciones de comportamiento y trastornos afectivos emocionales- el agresor y por último, los observadores (Carney y Merrel, 2001; Griffin y Gross, 2004; Olweus, 1993). Para Olweus (2001) los distintos roles que se pueden dar en una situación de acoso escolar constituyen el círculo del bullying y considera que todos los participantes van a verse afectados en un sentido negativo por esta situación.

La preocupación por el maltrato escolar ha dado lugar a numerosas intervenciones y acciones en diferentes países y en muy diferentes ámbitos (Martín, Fernández, Andrés, Del Barrio y Echeita, 2003). Para Benítez y Justicia (2006) en una fase inicial las investigaciones se han centrado junto a la definición del problema, en el análisis epidemiológico de la gravedad del fenómeno en cada contexto. Como resultado final de todas las investigaciones previas ha aumentado el desarrollo e implementación de programas y estrategias de prevención e intervención (Cowie y Olaffson, 2000; DíazAguado, 2005).

Para Garaigordobil y Oñederra (2010) se ha pasado en los últimos años de no intervenir a realizar cantidad de estudios y campañas preventivas en los centros. Entre las inter-

* Dirección para correspondencia [Correspondence address]: Benito León del Barco. Facultad Formación del Profesorado. Avd/ de la Universidad, s/n. 10007 Cáceres (España). E-mail: bleon@unex.es venciones centradas en el alumnado destacan las propuestas de ayuda entre iguales, que Cowie y Wallace (2000) clasifican en dos grupos: las que enfatizan el apoyo emocional y las que enfatizan la educación entre iguales como el aprendizaje cooperativo.

Olweus (1993) propone, entre otras medidas, para aplicar en el aula el uso del aprendizaje cooperativo. Para Avilés (2006) son los procedimientos de carácter cooperativo los que favorecen la convivencia, constituyendo un elemento preventivo de trabajo para evitar situaciones de acoso en el grupo. Díaz-Aguado (2006) señala que el aprendizaje cooperativo en grupos heterogéneos puede ser de gran eficacia para disminuir la exclusión y la violencia, mejorando la convivencia escolar en el aula.

Las investigaciones realizadas sobre los efectos del aprendizaje cooperativo en variables sociales, desde hace casi cuatro décadas, han encontrado que estas técnicas mejoran las relaciones interpersonales (Cook, 1978; Devries y Edwards, 1974 Díaz-Aguado, 2003; León, 2006; León, Felipe, Gozalo, Gómez y Latas, 2009; León, Gozalo y Polo, 2012; Ovejero, 1990; Slavin, 1983; Slavin y Cooper, 1999; Smith, Boulton y Cowie, 1993).

El aprendizaje cooperativo es una metodología que puede ayudar a resolver algunos de los problemas más acuciantes que existen en nuestras escuelas, incluido el acoso entre iguales.

Como señala Allport (1954) en su obra "Nature of prejudice" la convivencia de diversos grupos en un mismo lugar, por ejemplo el aula, es compleja y potencialmente conflictiva. Solamente habrá un cambio favorable en la atracción interpersonal, si el contacto entre miembros de grupos distintos estimula la interdependencia, la cooperación, la relación igualitaria entre ellos y el conocimiento de detalles sobre los demás. Las técnicas de aprendizaje cooperativo cum- 
plen con estas condiciones y sería una metodología esencial para mejorar las relaciones y la convivencia en el ámbito educativo. Johnson y Johnson (2000) defienden que el aprendizaje cooperativo es una herramienta eficaz para mejorar la convivencia, pues produce unas relaciones positivas entre los diversos grupos de estudiantes, proporciona una mayor atracción interpersonal y más apoyo social.

Cowie y Berdondini (2001) con una muestra de 117 alumnos de 8 a 11 años encontraron cambios en la expresión de emociones entre niños clasificados de víctimas, agresores y observadores después de trabajar en grupos cooperativos. $\mathrm{El}$ acoso escolar es un fenómeno en el que agresor, víctima y observadores, son partes integrantes de un mismo proceso. La intervención en aprendizaje cooperativo tiene que ir dirigida a todos los alumnos del aula, fomentando aquellas relaciones entre ellos, que impidan su aparición (Salmivalli y Peets 2009).

Cuando se habla de perfiles de la dinámica bullying se hace referencia a un conjunto de rasgos y características personales y sociales que diferencian a agresor, víctima y observadores, lo que ayuda a predecir comportamientos y a intervenir en las distintas formas de actuar de ciertos alumnos (Sánchez y Cerezo, 2011). Con esta investigación pretendemos determinar sobre qué perfil de la dinámica bullying (Agresor, víctima, Observador) es más relevante una intervención en aprendizaje cooperativo.

Para ello utilizaremos pruebas del tamaño de efecto, utilizando el estadístico $d$, propuesto por Cohen (1977) probablemente la prueba más empleada para calcular el tamaño del efecto. Las comparaciones de medias o los contraste de hipótesis presentan críticas y limitaciones, ya que en ocasiones no responden a las cuestiones cla magnitud de las diferencias es grande o pequeña? y ¿qué relevancia o importancia tienen? Puesto que se pueden hallar diferencias estadísticamente significativas pero pequeñas e irrelevantes y en otras ocasiones las diferencias pueden ser grandes e importantes, sin que sean significativas. Por lo que la consideración de la magnitud del efecto, puede usarse para decidir si los resultados son realmente pobres o, por el contrario, útiles e importantes (Kirk, 1996).

\section{Método}

\section{Participantes}

Se trabajó con dos grupos de participantes, un primer grupo que se utilizó para el análisis psicométrico del instrumento y un segundo grupo con el cual se llevó a cabo la intervención. El primer grupo se compuso de 311 alumnos, 160 cursaban $5^{\circ}$ de primaria y 151 cursaban $6^{\circ}$ de primaria. Con relación al género, 129 alumnos son mujeres y 182 son varones. La edad estaba comprendida entre los 10 y 12 años, siendo la media de 11.12. Del total de alumnos, 66 tenían 10 años, 147 tenían la edad de 11 años y 98 contaban con 12 años de edad. El sistema de elección fue de tipo aleatorio por conglomerados. Se seleccionaron al azar seis centros educativos de la provincia de Cáceres, cinco centros públicos y uno concertado.

El segundo grupo de participantes que sirve de muestra para la intervención estaba formado por 110 sujetos de 10 a 12 años. El $51.10 \%$ son mujeres y el $48.90 \%$ son varones. Participaron 68 alumnos de quinto curso de primaria y 42 de sexto de primaria. Fueron elegidos, igualmente, de forma aleatoria por conglomerados. Se seleccionaron al azar tres centros educativos públicos de la provincia de Cáceres, diferentes a los que, previamente, participaron en la cumplimentación de la escala. Uno de los centros es urbano (2 grupos de $5^{\circ}$ de primaria y dos grupos de $6^{\circ}$ de primaria) y dos son rurales (Un grupo de $5^{\circ}$ de primaria y $6^{\circ}$ cada centro). A los profesores que voluntariamente quisieron participar en la experiencia se les entrenó en un seminario de doce horas en aprendizaje cooperativo. La intervención en técnicas de aprendizaje cooperativo se desarrolló durante cuatro meses, desde febrero hasta mayo de 2008.

\section{Instrumentos}

Previo a la elaboración de los instrumentos se realizó una revisión de los cuestionarios y escalas ya existentes. Con respecto a las tres escalas de frecuencias de acoso escolar entre iguales, no se encontró ningún cuestionario que se ajustara exactamente al planteamiento de trabajo: ajustar al máximo las modalidades de conductas de agresión con las que se quería trabajar (Verbales y físicas directas e indirectas y de exclusión social), y someterlas por igual a las tres perspectivas del acoso escolar (agresor, víctima y observador), dejando abierto el escenario al que poder atribuir las distintas conductas de agresión.

Se elaboró la Escala de Frecuencia de Acoso Escolar entre Iguales para medir la frecuencia de las conductas de acoso escolar desde tres percepciones distintas: la visión del agresor, de la víctima y del observador. Las tres subescalas se presentan en formato Likert con cinco intervalos en forma numérica de 1 al 5, que representan un continuo que va desde "Nunca" hasta "Siempre". Hemos intentado que los ítems muestren adecuadamente los contenidos más relevantes del dominio que pretendemos evaluar. Siguiendo la definición clásica de Farrington (1993) y los trabajos de Del Barrio, Martín, Montero, Gutiérrez y Fernández (2003) hemos considerado conductas de maltrato directas (físicas y verbales) e indirectas, así como conductas de exclusión social.

\section{Escala de Frecuencia de Acoso Escolar entre Iguales. Perspectiva Agresor}

Tras la depuración de la escala (19 ítems), ésta quedó constituida finalmente por dieciséis ítems, con un Alfa de Cronbach de .894. Para calcular la validez de constructo del instrumento, se realizó un análisis factorial. La medida de adecuación muestral de Kaiser-Meyer-Olkin ofrece un valor de .914. La prueba de esfericidad de Bartlett resulta significativa (Chi-cuadrado $=1863.467$ gl. $=120$ y $p<.001)$. El valor 
de, KMO indica que la relación entre las variables es alta y Bartlett muestra la aplicabilidad del análisis factorial, por lo que tiene sentido realizar el análisis factorial.

Como podemos observar en la Tabla 1 los datos nos muestran la existencia de 3 factores que explican casi el 55\% de la varianza total de las escalas. El primer factor al que llamaremos "Agresiones verbales y físicas directaf" explica el $26.51 \%$ de la varianza y se refiere a conductas como: insultar, burlarse, despreciar, pegar, tirar y lanzar objetos a com- pañeros. La consistencia interna es aceptable, el alfa de Cronbach es de .880 .

El segundo factor "Agresiones de exclusión social" explica el $14.34 \%$ de la varianza y se refiere a conductas como: no dejar participar en juego, dejar solos, rechazar e ignorar a compañeros. Presenta un alfa de Cronbach de .636. El tercer factor "Agresiones verbales y físicas indirectas" explica el 14.10\% de la varianza y se refiere a conductas: contar historias falsas, chantajear y destrozar cosas de los compañeros. Presenta una consistencia interna de alfa igual a .631.

Tabla 1. Análisis factorial Escala de Frecuencia de Acoso Escolar entre Iguales. -Perspectiva Agresor. (Componentes principales con rotación Oblimín).

\begin{tabular}{|c|c|c|c|c|c|c|}
\hline Media & D. Tipica & Comu. & Ítems del instrumento depurados & Factor 1 & Factor 2 & Factor 3 \\
\hline 2.10 & .807 & .640 & 1. He insultado a otros compañeros & .725 & .338 & .165 \\
\hline 1.73 & 1.02 & .410 & 2. He despreciado y "he hecho de menos" a otros compañeros & .624 & .144 & -.006 \\
\hline 1.91 & .914 & .661 & 3. Me he burlado de otros compañeros & .727 & .324 & .165 \\
\hline 1.38 & .779 & .478 & 4. He amenazado a otros compañero & .573 & .086 & .376 \\
\hline 1.46 & .740 & .498 & 6. He dejado en ridículo a otros compañeros & .520 & .442 & .181 \\
\hline 1.75 & .910 & .626 & 7. He pegado a otros compañeros & .722 & .099 & .308 \\
\hline 1.40 & .722 & .570 & 8.He tirado las cosas de otros compañeros & .696 & .076 & .284 \\
\hline 1.84 & .879 & .575 & 9. He empujado a otros compañeros & .672 & .222 & .273 \\
\hline 1.46 & .823 & .506 & 10. He lanzado objetos a otros compañeros & .607 & .198 & .313 \\
\hline 1.14 & .489 & .579 & 13. He destrozado cosas de otros compañeros & .241 & .163 & .703 \\
\hline 1.41 & .762 & .350 & 14. No he dejado participar en actividades, juegos a otros & .313 & .452 & .219 \\
\hline 1.38 & .543 & .548 & 15. He dejado solos a otros compañeros & .128 & .723 & .092 \\
\hline 1.38 & .611 & .630 & 16. He rechazado a otros compañeros & .100 & .788 & .005 \\
\hline 1.60 & .679 & .496 & 17. He ignorado a otros compañeros & .311 & .589 & .229 \\
\hline 1.26 & .556 & .571 & 18.He inventado historias falsas de otros compañeros & .064 & .452 & .735 \\
\hline \multirow[t]{4}{*}{1.23} & .572 & .653 & 19. He chantajeado a otros compañeros & .300 & .034 & .750 \\
\hline & & & & Factor 1 & Factor 2 & Factor 3 \\
\hline & & & Porcentaje de varianza explicada (Total $54.95 \%$ ) & $26.51 \%$ & $14.34 \%$ & $14.10 \%$ \\
\hline & & & Alfa (Total .894) & .880 & .636 & .631 \\
\hline
\end{tabular}

Escala de Frecuencia de Acoso Escolar entre Iguales. Perspectiva Victima

Tras la depuración de la escala (19 ítems), ésta quedó constituida finalmente por dieciocho ítems, el Alfa de Cronbach nos da un valor de .916. Tanto la medida de adecuación muestral de Kaiser-Meyer-Olkin, que ofrece un valor de .929, como la prueba de esfericidad de Barlett (Chicuadrado $=2507.828 \mathrm{gl} .=153 \mathrm{y} p<.001)$ nos indican un perfecto ajuste de la muestra a la escala que estamos estudiando.

Como podemos observar en la Tabla 2 los datos nos muestran la existencia de 4 factores que explican casi el $62 \%$ de la varianza total de las escalas. El primer factor compuesto por 6 ítems lo hemos denominado "Sufrir agresiones verbales directas", incluye reactivos relacionados con ser insultado, menospreciado, ridiculizado, burlas, amenazas y ser blanco de críticas. Contribuye a explicar el $20.61 \%$ de la varianza y presenta una consistencia interna de alfa igual a 869 .

El segundo factor lo hemos denominado "Sufrir exclusión social" e incluye 5 elementos que recogen situaciones en las que los compañeros dejan al niño sólo, le rechazan, le ignoran, inventan historias sobre él o le hacen chantaje. De elevado poder explicativo, sobre el $18.11 \%$ de la varianza total. La consistencia interna es muy aceptable, el alfa de Cronbach es de .851 .
El tercer factor agrupa conductas de "Sufrir agresión física directa”. Las agresiones se refieren a situaciones en las que los compañeros tiran las cosas del alumno, le empujan, le lanzan objetos o directamente le pegan, estas agresiones físicas, estarían orientadas en ocasiones, a la exclusión del alumno/a víctima en la participación de actividades. Explica un 13.36\% de la varianza y presenta un alfa de Cronbach de .700. Por último, el cuarto factor, que agrupa un menor número de ítems, lo hemos denominado "Sufrir agresión física indirecta" y se refiere a aquellas situaciones en la que el alumno es víctima de robos y de destrozos en sus pertenencias. Explica un $9.75 \%$ de la varianza y presenta un alfa de Cronbach de .630 .

\section{Escala de Frecuencia de Acoso Escolar entre Iguales. Perspectiva Observador}

Tras la depuración de la escala (19 ítems), ésta quedó constituida finalmente por dieciséis ítems. Alfa de Cronbach de .921. La medida de adecuación muestral de Kaiser-MeyerOlkin ofrece un valor de .936. La prueba de esfericidad de Bartlett resulta significativa (Chi-cuadrado $=2367.777$ gl. $=$ 120 y $p$ <.001). Ambos valores. KMO y Bartlett, indican que tiene sentido realizar el análisis factorial.

Como podemos observar en la Tabla 3 los datos nos muestran la existencia de 4 factores que explican casi el 66\% 
de la varianza total. El primer factor al que llamaremos "Agresiones verbales y físicas directas" explica el 19.835\% de la varianza y se refiere a conductas observadas como: hablar mal, despreciar, burlarse, amenazar, Pegar, ridiculizar a compañeros. La consistencia interna es aceptable, el alfa de Cronbach es de .866 .

Tabla 2. Análisis factorial Escala de Frecuencia de Acoso Escolar entre Iguales. Perspectiva Victima. (Componentes principales con rotación Oblimín).

\begin{tabular}{|c|c|c|c|c|c|c|c|}
\hline Media & D. Tipica & Соти. & Ítems del instrumento depurados & Factor 1 & Factor 2 & Factor 3 & Factor 4 \\
\hline 2.53 & 1.12 & .553 & 1.Otros compañeros me han insultado & .703 & .102 & .184 & .119 \\
\hline 1.83 & .990 & .448 & 2. Me han despreciado. me "han hecho de menos" & .598 & .284 & .093 & .040 \\
\hline 2.15 & 1.00 & .693 & 3.Otros compañeros se han burlado de mí & .718 & .366 & .177 & .112 \\
\hline 1.57 & .837 & .590 & 4. Otros compañeros me han amenazado & .663 & .165 & .188 & .297 \\
\hline 2.10 & .969 & .637 & 5.Otros compañeros han hablado mal de mí & .624 & .436 & .170 & .171 \\
\hline 1.69 & .898 & .608 & 6.Otros compañeros me han dejado en ridículo & .650 & .329 & .212 & .180 \\
\hline 1.76 & .860 & .633 & 7.Otros compañeros me han pegado & .481 & .133 & 607 & .122 \\
\hline 1.64 & .832 & .556 & 8. Otros compañeros han tirado mis cosas & .170 & .154 & .683 & .193 \\
\hline 1.93 & .867 & .652 & 9.Otros compañeros me han empujado & .397 & .108 & .677 & .155 \\
\hline 1.61 & .864 & .664 & 10.Otros compañeros me han lanzado objetos & .100 & .199 & .778 & .097 \\
\hline 1.27 & .573 & .742 & 12. Otros compañeros me han robado & .199 & .010 & .099 & .832 \\
\hline 1.27 & .544 & .596 & 13. Otros compañeros han destrozado mis cosas & .208 & .306 & .192 & .650 \\
\hline 1.67 & .978 & .412 & 14. No me han dejado participar en actividades... & .241 & .264 & .406 & -.187 \\
\hline 1.62 & .869 & .715 & 15.Otros compañeros me han dejado solo & .300 & .783 & .102 & .041 \\
\hline 1.54 & .795 & .720 & 16.Otros compañeros me han rechazado & .399 & .741 & .098 & .048 \\
\hline 1.64 & .796 & .618 & 17.Otros compañeros me han ignorado & .255 & .666 & .320 & .088 \\
\hline 1.59 & .820 & .629 & 18.Han inventado historias falsas sobre mí & .138 & .623 & .137 & .451 \\
\hline \multirow[t]{4}{*}{1.36} & .678 & .667 & 19.Otros compañeros me han hecho chantaje & .182 & .631 & .330 & .356 \\
\hline & & & & Factor 1 & Factor 2 & Factor 3 & Factor 4 \\
\hline & & & Porcentaje de varianza explicada (Total 61.84\%) & $20.61 \%$ & $18.11 \%$ & $13.37 \%$ & $9.75 \%$ \\
\hline & & & Alfa (Total .916) & .869 & .851 & .700 & .627 \\
\hline
\end{tabular}

El segundo factor "Agresiones físicas indirectas" explica el $18.293 \%$ de la varianza y se refiere a conductas observadas como: tirar o destrozar cosas de otros compañeros, lanzar objetos o empujar sin provocar daños físicos en el agredido o como forma encubierta de agresión. Presenta un alfa de Cronbach de .790. El tercer factor "Agresiones de exclusión social' explica el $15.087 \%$ de la varianza y se refiere a conduc- tas observadas, como: no dejar participar en juego, dejar solos, rechazar a compañeros. Presenta una consistencia interna de alfa igual a .831. El cuarto factor "Agresiones verbales indirectas" explica el $12.924 \%$ de la varianza y se refiere a conductas observadas como: contar historias falsas y chantajear a otros compañeros. Presenta una consistencia interna de alfa igual a .668 .

Tabla 3. Análisis factorial Escala de Frecuencia de Acoso Escolar entre Iguales. Perspectiva Observador. (Componentes principales con rotación Oblimín).

\begin{tabular}{|c|c|c|c|c|c|c|c|}
\hline Media & D. Tipica & Comu & Ítems del instrumento depurados & Factor 1 & Factor 2 & Factor 3 & Factor 4 \\
\hline 2.17 & 1.000 & .609 & 2. He presenciado desprecios & .724 & .144 & .247 & .202 \\
\hline 2.61 & 1.082 & .651 & 3. He presenciado cómo se han burlado de otros & .710 & .309 & .210 & .092 \\
\hline 2.07 & 1.115 & .692 & 4. He presenciado amenazas a otros compañeros & .688 & .366 & .023 & .291 \\
\hline 2.67 & 1.133 & .652 & 5. He oído hablar mal de otros compañeros & .727 & .144 & .247 & .202 \\
\hline 2.17 & 1.071 & .632 & 6. He presenciado como ridiculizaban a los demás & .562 & .442 & .334 & .100 \\
\hline 2.59 & 1.153 & .710 & 7. He presenciado como pegaban a otros compañeros & .595 & .531 & .263 & -.069 \\
\hline 2.16 & 1.109 & .648 & 8. He presenciado como tiraban las cosas de otros & .172 & .714 & .178 & .271 \\
\hline 2.43 & 1.147 & .629 & 9. He visto como empujaban a otros & .322 & .597 & .398 & .106 \\
\hline 2.08 & 1.090 & .618 & 10. He presenciado como lanzaban objetos a otros & .149 & .752 & .118 & .128 \\
\hline 1.46 & .779 & .576 & 13. He visto como destrozaban cosas de otros & 205 & .670 & .159 & .244 \\
\hline 2.10 & 1.044 & .714 & 14. He visto como no dejaban participar a otros & .211 & .204 & .789 & .070 \\
\hline 2.00 & 1.035 & .704 & 15. He presenciado como dejaban solos a otros & .179 & .202 & .743 & .282 \\
\hline 1.91 & 1.003 & .676 & 16. He presenciado como rechazaban a otros & .232 & .225 & .584 & .481 \\
\hline 2.05 & 1.060 & .684 & 17. He visto como ignoraban a otros & .241 & .177 & .544 & .346 \\
\hline 1.88 & 1.022 & .702 & 18. He oído historias falsas de otros compañeros & .173 & .130 & .220 & .779 \\
\hline \multirow[t]{4}{*}{1.77} & .970 & .685 & 19. He visto como chantajeaban a otros & .238 & .372 & .132 & .688 \\
\hline & & & & Factor 1 & Factor 2 & Factor 3 & Factor 4 \\
\hline & & & Porcentaje de varianza explicada (Total $66.138 \%$ ) & $19.83 \%$ & $18.29 \%$ & $15.08 \%$ & $12.92 \%$ \\
\hline & & & Alfa (Total .921) & .866 & .790 & .831 & .668 \\
\hline
\end{tabular}

Para Henson y Roberts (2006) una buena práctica para el estudio psicométrico de un cuestionario es confirmar la es- tructura factorial encontrada en el análisis exploratorio con un análisis factorial confirmatorio. Es necesario para poder 
realizar las estimaciones utilizando el método de Máxima Verosimilitud (Jöreskog y Sörbom, 1996) que se cumplan los supuestos de linealidad y que todas las variables observadas incluidas en el modelo sigan una distribución normal. Los gráficos de dispersión de los residuos realizados reflejaron que existe linealidad entre las variables estimadas.

Previo al análisis para determinar si la muestra cumple o no con el criterio de normalidad, procedimos a la detección de los valores atípicos aplicando la denominada distancia de Mahalanobis, mediante la opción Tests for normality and outliers del programa AMOS. No obstante, después de eliminar las puntuaciones atípicas, los datos de la muestra no cumplían con el criterio de normalidad. Por ello, utilizamos como método alternativo para la estimación de los parámetros el método de Mínimos Cuadrados Ponderados (Weighted Least Squares) que se encuentra incluido en el método de estimación de Distribución Libre Asintótica (ADF) en el programa AMOS.

Para determinar si el modelo se ajusta adecuadamente a los datos, hemos utilizado los siguientes índices de bondad de ajuste: Probabilidad chi cuadrado ( $\chi 2)$, indica el parecido entre las covarianzas observadas con aquellas que se encuentran en el modelo hipotético, valores no significativos (mayores a .05) indican una correspondencia aceptable entre el modelo propuesto y los datos. El chi cuadrado dividido por los grados de libertad (CMIN/DF), este índice permite comprobar, de forma más real, el nivel de ajuste entre la solución propuesta y los datos empíricos, es un índice menos sensible al tamaño de la muestra, de modo que valores por debajo de 2 son considerados como indicadores de un buen ajuste y por debajo de 5 son considerados como aceptables (Hu y
Bentler, 1999). El indice CFI (Comparative Fit Index), su valor oscila entre 0 y 1 , se considera que el modelo ajusta cuando el valor es mayor de .90. Es uno de los índices de ajuste más empleados. El indice TLI (Tuker-Lewis Index), al igual que el CFI toma valores entre 0 y 1 , siendo aceptables valores mayores de .90 (Bentler, 1995). Índice RMSEA (Roat Mean Square Error of Aproximation), valores por debajo de .08 se consideran aceptables.

En la Tabla 4 mostramos los estadísticos de bondad de ajuste considerando en cada escala dos modelos: factores independientes y factores correlacionados. Descartamos en las tres escalas los modelos de factores independientes porque todos los indicadores obtienen valores alejados de los límites aceptados y nos permiten afirmar que el modelo de factores independientes no presenta un buen ajuste. Mejor ajuste, presentan los modelos de factores relacionados aunque sin llegar a ser optimo en el caso de agresor y víctima, lo que justifica el análisis factorial con rotación Oblimín. En ninguno de los tres modelos de factores relacionados se obtiene un valor $\chi^{2}$ significativo $(p>.05)$. El valor de $\chi^{2}$ suele estar influido por el tamaño de la muestra y supone una hipótesis excesivamente restrictiva, el índice $C M I N / D F$, menos sensible al tamaño de la muestra, permite comprobar, de forma más real, el nivel de ajuste entre la solución propuesta y los datos empíricos. Este índice presenta un buen ajuste en los modelos de factores relacionados de las escala Observador y Víctima y en menor medida en la escala Agresor. Igualmente, los indicadores de ajuste CFI. TLI y RMSEA presentan valores óptimos en la escala Observador y Víctima, presentando problemas de ajuste la escala Agresor.

Tabla 4. Índices de bondad de ajuste de los modelos propuestos.

\begin{tabular}{ccccccc}
\hline & Escalas Acoso Escolar & $\chi 2$ & CMIN/DF & CFI & TLI & RMSEA \\
\hline \multirow{2}{*}{ Agresor } & 3 factores independientes & $p<.001$ & 6.957 & .642 & .599 & .139 \\
& 3 factores relacionados & $p<.001$ & 5.159 & $\mathbf{. 7 5 7}$ & $\mathbf{. 7 2 0}$ & .116 \\
Víctima & 4 factores independientes & $p<.001$ & 6.211 & .709 & .677 & .130 \\
& 4 factores relacionados & $p<.001$ & 3.327 & $\mathbf{. 8 7 6}$ & $\mathbf{. 8 5 6}$ & .080 \\
\multirow{2}{*}{ Observador } & 4 factores independientes & $p<.001$ & 7.603 & .693 & .656 & .146 \\
& 4 factores relacionados & $p<.001$ & 2.522 & $\mathbf{. 9 3 3}$ & $\mathbf{. 9 2 1}$ & .060 \\
\hline
\end{tabular}

Para tratar de verificar la estructura factorial teorizada a partir de los diferentes ítems hemos utilizado el método de codificación de efectos propuesto por Litte. Slegers y Card (2006), encontrando los siguientes valores en las cargas factoriales:

1. Escala de Frecuencia de Acoso Escolar entre Iguales. Perspectiva Agresor: Factor 1 (Agresiones verbales y fisicas directas), valores que oscilan entre $\lambda=.719$ y $\lambda=.907$; Factor 2 (Agresiones de exclusión social), valores entre $\lambda=.708$ y $\lambda=.852$ y Factor 3 (Agresiones verbales $y$ físicas indirectas), valores entre $\lambda=$ .777 y $\lambda=.940$. Los indicadores de las variables latentes muestran cargas factoriales $>.700$, esto indica que las variables latentes están bien definidas y. por tanto, la forma en que se han evaluado los factores es adecuada.

2. Escala de Frecuencia de Acoso Escolar entre Iguales. Perspectiva Victima: Factor 1 (Sufrir agresiones verbales), valores que os- cilan entre $\lambda=.659$ y $\lambda=.855$; Factor 2 (Sufrir exclusión social), valores entre $\lambda=.678$ y $\lambda=.795$; Factor 3 (Sufrir agresión fisica directa), valores entre $\lambda=.731$ y $\lambda=.878 \mathrm{y}$ Factor 4 (Sufrir agresión física indirecta), valores entre $\lambda=$ .277 y $\lambda=.752$. A excepción de este último factor, cuyo valor es imperceptible, las cargas factoriales $>.650$ indican que el resto de los factores está adecuadamente evaluados.

3. Escala de Frecuencia de Acoso Escolar entre Iguales. Perspectiva Observador: Factor 1 (Agresiones verbales y físicas directas), valores que oscilan entre $\lambda=.773$ y $\lambda=.830$; Factor 2 (Agresiones físicas indirectas), valores entre $\lambda=.701 \mathrm{y} \lambda=$ .819; Factor 3 (Agresiones de exclusión social), valores entre $\lambda$ $=.715$ y $\lambda=.842$ y Factor 4 (Agresiones verbales indirectas), valores entre $\lambda=.800$ y $\lambda=.808$. Los indicadores de las variables latentes muestran cargas factoriales $>.700$, la 
forma en que se han evaluado los factores de esta escala es correcta.

\section{Diseño}

Hemos utilizado una metodología cuasi-experimental. Las dos estrategias fundamentales para paliar los defectos de la metodología cuasi-experimental son: 1. la inclusión de un grupo control. 2. Tomar una medida tras la aplicación del tratamiento y otra previa al mismo. Hemos aplicado un diseño intergrupo pretest-postest con grupo control no equivalente.

Se ha trabajado con grupos independientes, un grupo experimental, formado por 45 alumnos de $5^{\circ}$ de primaria $y$ 13 alumnos de $6^{\circ}$ de primaria distribuidos en cuatro aulas pertenecientes a tres centros y un grupo control, formado por 23 alumnos de $5^{\circ}$ de primaria y 29 de $6^{\circ}$ de primaria distribuidos, igualmente, en cuatro aulas de los centros participantes. Existe una condición experimental, intervención con técnicas de aprendizaje cooperativo (Jigsaw). El grupo control no recibe la intervención y ambos grupos, experimental y control, cumplimentan en fase pretest y postest la Escala de Frecuencia de Acoso Escolar entre Iguales (Agresor. Victima y Observador).

\section{Resultados}

Sometimos los datos a la prueba de Kolmogorov-Smirnov para analizar la distribución normal y determinar el uso de pruebas paramétricas o no paramétricas en la comparación de medias relacionadas (pretest-postest) e independientes (grupo experimental-grupo control). En la Tabla 5 presentamos los resultados obtenidos, hemos encontrado diferencias significativas debidas a la intervención en técnicas de aprendizaje cooperativo en la perspectiva Agresor en los factores: "Agresiones verbales y físicas directas" y "Agresiones de exclusión social". Para la perspectiva Víctima: "Sufrir agresión verbal". "Sufrir exclusión social" y "Sufrir agresiones físicas directas". En la perspectiva Observador: "Observar agresiones verbales y físicas directas". "Observar agresiones físicas directas" y "Observar agresiones de exclusión social"

Para mejorar y completar la información que aporta la aplicación de las pruebas de significación y determinar sobre qué perfil de la dinámica bullying ha sido más eficaz la intervención, hemos calculado el tamaño del efecto producido intragrupo (prestes-postest) e intergrupo (experimentalcontrol) utilizando el estadístico $d$. propuesto por Cohen (1977), probablemente la prueba más empleada para calcular el tamaño del efecto (Tabla 5). Los tamaños del efecto encontrados (entre $d=0.20$ y $d=0.36$ ) son medios en las comparaciones intragrupo para el grupo experimental (pretest-postest) y muy bajos $(d \leq 0.09)$, casi irrelevantes en el grupo control. Por otro lado, en la comparación postest intergrupo (experimental-control) el tamaño del efecto ha sido elevado para el factor de exclusión social para los perfiles observador $(d=0.64)$ y agresor $(d=0.65)$. También, obtiene un tamaño elevado el factor agresiones verbales y físicas directas para el perfil observador $(d=0.57)$. Mientras que el tamaño del efecto ha sido más bajo en el perfil víctima.

Tabla 5. Pruebas estadísticas contraste de medias, tamaño del efecto (d de Cohen) y binomial effect size display (BESD).

\begin{tabular}{|c|c|c|c|c|c|c|c|}
\hline \multirow[t]{2}{*}{ Factores Escala Acoso Escolar } & \multicolumn{2}{|c|}{$\begin{array}{l}\text { Grupo Experimental } \\
\text { (pretest-postest) }\end{array}$} & \multicolumn{2}{|c|}{$\begin{array}{l}\text { Grupo Control } \\
\text { (pretest-postest) }\end{array}$} & \multicolumn{3}{|c|}{$\begin{array}{c}\text { Grupos } \\
\text { (Experimental-Control) }\end{array}$} \\
\hline & $t / Z$ & $d$ & $t / Z$ & $d$ & $t / U$ & $d$ & $\%$ Éxito \\
\hline Realizar agresiones verbales y físicas directas ${ }^{1}$ & $1.947^{*}$ & .20 & .386 & .04 & $-2.01 *$ & .39 & $59.5 \%$ \\
\hline Realizar agresiones de exclusión social ${ }^{2}$ & $-2.140 *$ & .30 & -.911 & -.09 & $1028^{* *}$ & .65 & $65.5 \%$ \\
\hline Sufrir agresión verbal ${ }^{2}$ & $-2.125^{*}$ & .22 & -.025 & -.04 & 1388 & .09 & $52 \%$ \\
\hline Sufrir exclusión social ${ }^{2}$ & $-2.397 *$ & .30 & -.287 & .08 & 1429 & .10 & $52.5 \%$ \\
\hline Sufrir agresiones físicas directas ${ }^{2}$ & $-2.369 *$ & .34 & -1.512 & .16 & 1497 & .04 & $51 \%$ \\
\hline Observar agresiones verbales y físicas directas ${ }^{1}$ & $1.846^{*}$ & .26 & .111 & .01 & $-3.103^{* *}$ & .57 & $63.5 \%$ \\
\hline Observar agresiones físicas indirectas ${ }^{2}$ & $-2.676^{* *}$ & .34 & -.666 & .07 & $1233^{*}$ & .39 & $59.5 \%$ \\
\hline Observar agresiones de exclusión social ${ }^{1}$ & $2.518^{*}$ & .36 & -0.047 & -.01 & $-3.423^{* *}$ & .64 & $65 \%$ \\
\hline
\end{tabular}

${ }^{1}$ Prueba paramétrica t de Student (t)

${ }^{2}$ Muestras relacionadas, prueba no paramétrica Rangos de Wilcoxon (Z). Muestras Independientes prueba no paramétrica U de Mann-Whitney (U)

$* p<.05 \quad * * p<.01$

Por último, para poder interpretar la efectividad de la intervención calculamos el BESD (Binomial Effect Size Display) que nos permite confeccionar una tabla de éxitos y fracasos. Para el grupo experimental obtuvimos un \% de éxito del 65 en el factor exclusión social para los perfiles agresor y observador. En definitiva el grupo que recibió la intervención consigue un $30 \%$ más de eficacia que el grupo control sobre el factor "Agresiones de exclusión social".

\section{Discusión}

En primer lugar, nuestros resultados indican que se ha producido una reducción significativa del acoso escolar (agresiones de exclusión social y agresiones verbales y físicas directas) como consecuencia de la intervención en aprendizaje cooperativo. Con nuestro trabajo disponemos de más datos sobre cómo operan las técnicas de aprendizaje cooperativo sobre el acoso entre iguales. 
En la técnica de aprendizaje cooperativo utilizada cada alumno recibe un trozo de un tema que tiene que leer, estudiar y preparar. A continuación, los alumnos de los diferentes grupos que tienen el mismo fragmento se reúnen en "grupos de expertos" para discutir y profundizar en la información que les corresponde. Finalmente, cada estudiante vuelve a su grupo y enseña al resto de compañeros lo que ha aprendido. Esta técnica posibilita un contacto personal puesto que se generan muchas oportunidades de interacción y aumento de la empatía.

Como afirman Ramírez y Rodríguez (2006) y León. Felipe y Gómez (2010) el hecho de que los alumnos estén en el aula, y por tanto exista sólo un contacto presencial, no garantiza una mejora de las relaciones intergrupales. Distintos estudios como los de Cook (1985); Stephan (1987), o más recientemente el de Paluck y Green (2009) demuestran que para que el contacto sea efectivo debe tratarse de un contacto directo y personal que facilite la percepción de las características individuales de los demás, en este sentido, Pérez y Garaigordobil (2004) afirman que la interacción entre iguales y en concreto, las dinámicas socio-afectivas en el aula, puede desempeñar un importante papel afectando no sólo a los procesos de socialización, sino también los procesos de desarrollo cognitivo, afectivo y emocional. La mejora de la convivencia se puede lograr mediante contactos en condiciones de igualdad y buscando objetivos comunes mediante la cooperación (Torrego, 2012). En definitiva, los participantes se han beneficiado de un contacto directo y personal motivado por las técnicas de aprendizaje cooperativo que han mejorado sus relaciones.

Según Díaz-Aguado (2003), con los procedimientos educativos tradicionales las oportunidades se distribuyen de forma desigual en el aula, dando lugar a la exclusión e intolerancia entre los alumnos. La técnica de aprendizaje cooperativo que hemos llevado a cabo en el aula ha contribuido a desarrollar oportunidades de igualdad de estatus que probablemente han permitido establecer relaciones de amistad entre todos los alumnos.

En palabras de Aronson ${ }^{1}$ y Osherow (1980), la técnica Jigsaw incrementa la atracción de los estudiantes hacia sus compañeros, disminuye la competitividad y aumenta la capacidad para ponerse en el lugar o papel de otra persona. La empatía nos hace ser más sensibles a las necesidades y demandas de nuestros compañeros. Además, se relaciona con la solución constructiva de los conflictos y las actitudes positivas hacia los demás (Garaigordobil y Maganto, 2011). De la misma manera, la cooperación se relaciona con la prevención de los conflictos y las actitudes positivas hacia los demás, como expone Díaz-Aguado (2005).

En segundo lugar, los tamaños del efecto intragrupo (prestest-postest) e intergrupo (experimental-control) indican que las diferencias significativas encontradas en su mayoría son relevantes. En el contexto de la investigación educacional suelen encontrarse valores más bajos que en otras

1 Autor de la Técnica Jigsaw. disciplinas. Cuando se trata de la aplicación de metodologías innovadoras, valores entre $d=0.30$ y $d=0.33$ son considerados relevantes (Valentine y Cooper, 2003; Borg. Gall, y Gall, 1993). Hattie (2009) encuentra un valor medio del tamaño de efecto de $d=0.40$ en un análisis de 500.000 intervenciones en el contexto educativo y considera que tamaños del efecto superiores a $d=0.60$ deben estimarse grandes. Johnson, Johnson y Stanne (2000) encuentran en una síntesis de 158 estudios sobre aprendizaje cooperativo un valor medio $d=0.45$ cuando comparan la cooperación frente a la competición en el rendimiento académico y un $d=0.51$ cuando la comparación se realiza frente al trabajo individual. Cuando se trata de mejorar la calidad de las relaciones sociales entre compañeros la cooperación obtiene frente a la competición un valor de 0.68 y de 0.55 cuando se compara con el trabajo individual (Johnson. Johnson y Smith, 1998).

Nuestros resultados manifiestan que el tamaño del efecto intergrupo ha sido elevado para el factor de exclusión social para los perfiles observador $(d=0.64)$ y agresor $(d=0.65)$. También, obtiene un tamaño elevado el factor agresiones verbales y físicas directas para el perfil observador $(d=0.57)$. En síntesis tras el análisis del tamaño del efecto podemos afirmar que el aprendizaje cooperativo es eficaz sobre el perfil agresor y especialmente, sobre el observador. Este hecho es importante, pues en la mayoría de las investigaciones sobre acoso escolar se concluye que los porcentajes de testigos son más elevados que los de víctimas y agresores, un mismo caso de agresión puede ser observado por varios testigos (Avilés y Monjas, 2005; Benítez y Justicia, 2006; Defensor del Pueblo, 2006; León, Felipe, Gómez y López. 2011; Serrano e Iborra, 2005). Existen muchas investigaciones que han demostrado la relación positiva entre exposición a la violencia en el contexto escolar y el desarrollo de conductas agresivas (Cerezo, 2009; Flannery, Wester y Singer. 2004; Garaigordobil y Oñederra, 2008).

Las principales limitaciones de la investigación -además de las limitaciones propias de toda investigación cuasiexperimental y las derivadas de la utilización de autoinformes como método recogida de información-, están relacionas con las dificultades encontradas para lograr la colaboración del profesorado de los centros que fueron escogidos, lo que limitó el tiempo de duración de la intervención, siendo éste un factor importante en la eficacia de los programas contra el bullying (Díaz-Aguado, 2005), e impidió el acceso a un mayor tamaño muestral, condicionando negativamente la posible generalización de los resultados. Esto hace patente la necesidad de incidir en mostrar al profesorado las ventajas de trabajar con técnicas de aprendizaje cooperativo de manera general y en particular en relación con el Bullying. A pesar de estas limitaciones, este tipo de investigación es un recurso necesario, que aporta información en contextos reales de la vida, desprovistos de situaciones artificiales.

En líneas futuras de investigación, habría que ordenar y determinar, según el valor del tamaño del efecto, qué técnicas de aprendizaje cooperativo son más relevantes para propiciar un cambio que disminuya la violencia. Algunas inves- 
tigaciones como las de Slavin (1983) obtienen resultados más positivos con las técnicas de aprendizajes por equipos (Student Team learning) que con las técnicas de aprendiendo juntos (Learning Together). Nuestra investigación coincide con otros estudios que han demostrado la eficacia del método Jigsaw sobre variables sociales (Goikoetxea y Pascual, 2002; Walter y Crogan, 1998). En el metanálisis efectuado por Johnson. Johnson y Stanne (2000) la técnica Jigsaw obtiene valores de tamaño del efecto medios y bajos cuando se compara con la competición $(d=0.29)$ y el trabajo individual $(d=0.13)$ en rendimiento. Este hecho nos lleva a pensar que los métodos de aprendizaje cooperativo se comportan de manera diferente cuando actúan sobre variables académicas, sociales o afec-

\section{Referencias}

Allport. G.W. (1954). The nature of prejudice. Cambridge. MA: AddisonWesley. (Trad. cast.: La naturaleza del prejuicio. Buenos Aires: Universitaria de Buenos Aires. 1962).

Aronson. E. y Osherow. N. (1980). Cooperation, prosocial behavior, and academic performance. En L. Bickman (Ed.), Applied social psychology annual, (Vol. 1, pp. 163-196). Beverly Hills: Sage.

Avilés. J. M. (2004). ¿Cómo abordar un caso de bullying? En R. Belandia (coord.) IV Curso de verano. Prevención de riesgos laborales en la enseñanza. (pp. 143-173). Bilbao: Instituto Vasco de Ergonomía y Stee-eilas.

Avilés. J. M. (2006). Bullying: El maltrato entre iguales. Agresores, victimas y testigos en la escuela. Salamanca: Amarú.

Avilés. J. y Monjas. I. (2005). Estudio de la incidencia de la intimidación y el maltrato entre iguales en la Educación Secundaria Obligatoria mediante el cuestionario CIMEI. Anales de Psicología, 21(1), 27-41.

Benítez. J. L. y Justicia. F. (2006). El maltrato entre iguales: descripción del fenómeno. Electronic Journal of Research in Educational Psychology, 9, 151170 .

Bentler. P. M. (1995). EQS structural equation program manual. Los Ángeles. CA: BMDP Statistical Software.

Borg. W., Gall. J. y Gall. M. (1993). Applying educational researcb: A practical guide. New York: Longman.

Carney. A. G. y Merrell. K. W. (2001). Bullying in schools: Perspectives on understanding and preventing an international problem. School Psychology International, 22(3), 364-382.

Cerezo. F. (2006). Violencia y victimización entre iguales. El bullying: estrategias de identificación y elementos para la intervención a través del Test Bull-S. Revista Electrónica de Investigación Psicoeducativa, 9, 333-352.

Cerezo. F. (2009). Bullying: análisis de la situación en las aulas españolas. International Journal of Psychology and Psychological Therapy, 9, 367-378.

Cohen. J. (1977). Statistical power analysis for the behavioral sciences. Nueva York: Academia Press.

Cook. S. W. (1978). Interpersonal and attitudinal outcomes in cooperating interracial groups. Journal of Research and Development in Education, 12, 27113.

Cook. S. W. (1985). Experimenting on social issues: The case of school desegregation. American Psychologist, 40, 452-460.

Cowie. H. y Berdondini. L. (2001). Children's reactions to cooperative group work: a strategy for enhancing peer relationships among bullies, victims and bystanders. Learning and Instruction, 11, 517-530.

Cowie. H. y Olafsson. R. (2000). The role of peer support in helping the victims of bullying in a school with high levels of aggression. School Psychology International, 21(1), 79-95.

Cowie. H. y Wallace. P. (2000). Peer support in action. London: Sage.

Defensor del Pueblo (2006). Violencia escolar: el maltrato entre iguales en la Educación Secundaria Obligatoria (1999-2006). Madrid: Publicaciones del Defensor del Pueblo.

Del Barrio. C., Martín. E., Montero. I., Gutiérrez. H. y Fernández. I. (2003). La realidad del maltrato entre iguales en los centros de secundaria españoles. Infancia y Aprendizaje, 26(1), 25-47. tivas y que habría que encontrar mediante pruebas de tamaño del efecto cuáles son las más relevantes en cada conjunto de variables.

Por último, pensamos en la utilidad de un trabajo en aprendizaje cooperativo en el aula para favorecer la convivencia y disminuir la violencia. Es innegable el hecho de que ser víctima escolar provoca alteraciones de comportamiento y trastornos afectivos emocionales. El aprendizaje cooperativo es una metodología innovadora que puede ayudar a resolver el problema del maltrato entre iguales por abuso de poder en el ámbito escolar influyendo sobre el agresor y el observador.

Devries. D. L y Edwards. K. J. (1974). Learning games and student teams: Their effects on classroom process. American Educational Research Journal, 4, 307-318.

Díaz-Aguado. M. J. (2003). Educación interculural y aprendizaje cooperativo. Madrid: Pirámide.

Díaz-Aguado. M. J. (2005). La violencia entre iguales en la adolescencia y su prevención desde la escuela. Psicothema, 17, 549-558.

Díaz-Aguado. M. J. (2006). Del acoso escolar a la cooperación en las aulas. Madrid: Pearson Educación.

Farrington. D. P. (1993). Understanding and preventing bullying. Crime and Justice: Review of Research, 17, 381-458

Flannery. D. J., Wester. K. L. y Singer. M. I. (2004). Impact of exposure to violence in school on child and adolescent mental health and behavior. Journal of Community Psychology, 32, 559-573.

Garaigordobil. M. y Oñederra. J. A. (2008). Bullying: incidence of peer violence in the schools of the Autonomous Community of the Basque Country. International Journal of Psychology and Psychological Therapy, 8, 5162.

Garaigordobil. M. y Oñederra. J. A. (2010). Un estudio comparativo de las conductas de acoso escolar percibidas por los directivos de los centros educativos y por los estudiantes de 10 a 16 años. En J. J. Gázquez \& M.C. Pérez (Eds.), Investigación en convivencia escolar: variables relacionadas (pp. 97-104). Granada: GEU.

Griffin. R. y Gross A. (2004). Childhood bullying: current empirical findings and future directions for research. Aggressive and violent behaviour, 9, 379400.

Hattie. J. (2009). Visible learning: A synthesis of meta-analyses in education. London: Routledge.

Henson. R. K. y Roberts. J. K. (2006). Use of Exploratory Factor Analysis in Published Research: Common Errors and Some Comment on Improved Practice. Educational and Psychological Measurement, 66, 393-416.

Hu, L., y Bentler, P. M. (1999). Cutoff criteria for fit indexes in covariance structure analysis: Conventional criteria versus new alternatives. Structural Equation Modeling: A Multidisciplinary Journal, 6(1), 1-55.

Johnson. D. W. y Johnson. R. (2000). The three Cs of reducing prejudice and discrimination. En S. Oskamp (Ed.), Reducing prejudice and discrimination (pp. 239-268). Mahwah. NJ: Lawrence Erlbaum Associates Publishers.

Johnson. D. W., Johnson. R. y Smith. K. A. (1998). Cooperative learning returns to College: What evidence is there that it works? Change. july/August, 27-35

Johnson. D. W., Johnson. R. y Stanne. M. (2000). Cooperative learning methods: A meta-analysis. University of Minnesota. Minneapolis: Cooperative Learning Center. 2000; http://mww.cooperation.org/pages/clmethods.html.

Jöreskog. K. G. y Sörbom. D. (1996). Lisrel 8: User's reference guide. Chicago: SSI Inc.

Kirk, R. E. (1996). Practical significance: A concept whose time has come. Educational \& Psychological Measurement, 56, 746-759. 
León. B. (2006). Elementos mediadores en la eficacia del aprendizaje cooperativo: Entrenamiento previo en habilidades sociales y dinámica de grupos. Anales de Psicología, 22, 105-112

León. B., Felipe. E. y Gómez. T. (2010). Variables individuales que influyen en las actitudes hacia la inmigración. Anales de Psicología, 26(2), 359-369

León. B., Felipe. E., Gómez. T. y López. V. (2011). Acoso escolar en la Comunidad de Extremadura vs. Informe español del Defensor del Pueblo (2006). Electronic Journal of Research in Educational Psychology, 9(2), 565-586.

León. B., Felipe. E., Gozalo. M., Gómez. T. y Latas. C. (2009). Mejora de las actitudes de los escolares hacia los alumnos inmigrantes mediante el aprendizaje cooperativo. Revista de Psicología General y Aplicada, 9, 159173.

León. B., Gozalo. M. y Polo M. I. (2012). Aprendizaje cooperativo y acoso entre iguales. Infancia y Aprendizaje, 35(1), 23-35.

Litte. T. D., Slegers. D. W. y Card. N. A. (2006). A non-arbitrary method of identifying and scaling latent variables in SEM and MACS models. Structural Equation Modeling, 13, 59-72.

Martín. E., Fernández. I., Andrés. S., Del Barrio. C. y Echeita. G. (2003). La intervención para la mejora de la convivencia en los centros educativos: modelos y ámbitos. Infancia y Aprendiraje, 26, 79-95.

Olweus. D. (1993). Bullying at school: What we know and what we can do. Oxford: Blackwells. Trad. cast. Conductas de acoso y amenaza entre escolares. Madrid: Morata. 1998.

Olweus. D. (2001). Peer harassment: a critical analysis and some important issues. En J. Juvonen y S. Graham (Eds.). Peer harassment in school: the plight of the vulnerable and victimized. (pp: 3-20). New York: Guilford Press.

Ovejero. A. (1990). El aprendizaje cooperativo. Barcelona: PPU.

Paluck. E. L., y Green. D. P. (2009). Prejudice reduction: What works? A review and assessment of research and practice. Annual review of psychology, 60, 339-367.
Ramírez. M. C. y Rodríguez. A. (2006). Variables predictoras de la actitud hacia los inmigrantes en la Región de Murcia (España). Anales de Psicología, 22, 76-80.

Salmivalli. C., y Peets. K. (2009). Bullies, victims, and bullyvictimrelationships. In K. Rubin. W. Bukowski \& B. Laursen (Eds.), Handbook of peer interactions, relationships, and groups (pp. 322-340). New York: Guilford.

Sánchez, C. y Cerezo, F. (2011). Estatus social de los sujetos implicados en bullying. Elementos para la mejora de la convivencia en el aula. Revista Española de Orientación y Psicopedagogía, 22(2), 137-149.

Serrano. A. e Iborra. I. (2005). Violencia entre compañeros en la escuela. Centro Reina Sofía para el Estudio de la Violencia.

Slavin. R. E. (1983). Cooperative Learning. New York: Longman. (Trad. cast. La enseñanza y el método cooperativo. México. Edamex 1985).

Slavin. R. E. y Cooper. R. (1999). Improving intergroup relations: lessons learned from cooperative learning programs. Journal of Social Issues, 55 , 647-663.

Smith. P. K., Boulton. M. J. y Cowie. H. (1993). The impact of cooperative group work on ethnic relactions in middle school. School Psychology International, 14, 21-42.

Stephan. W. (1987). The contact hypothesis in intergroup relations. Review of Personality and Social Psychology, 9, 13-40.

Torrego, J. C. (2012). La ayuda entre iguales para mejorar la convivencia escolar: Manual para la formación de alumnos/as ayudantes. Madrid: Narcea.

Valentine. J. y Cooper. H. (2003). Effect Size Substantive Interpretation Guidelines: Issues in the Interpretation of Effect Sizes. Washington. D.C.: What Works Clearing House.

Walker. I. y Crogan. M. (1998). Academic performance, prejudice, and the jigsaw classroom: New pieces to the puzzle. Journal of Community and Applied Social Psychology, 8, 381-393.

(Articulo recibido: 24-09-2013; revisado: 15-01-2015; aceptado: 22-01-2015) 